\title{
Ankle voluntary movement enhancement following robotic-assisted locomotor training in spinal cord injury
}

\author{
Deborah Varoqui ${ }^{1,2}$, Xun Niu ${ }^{1,2}$ and Mehdi M Mirbagheri ${ }^{1,2^{*}}$
}

\begin{abstract}
Background: In incomplete spinal cord injury (iSCl), sensorimotor impairments result in severe limitations to ambulation. To improve walking capacity, physical therapies using robotic-assisted locomotor devices, such as the Lokomat, have been developed. Following locomotor training, an improvement in gait capabilities—characterized by increases in the over-ground walking speed and endurance-is generally observed in patients. To better understand the mechanisms underlying these improvements, we studied the effects of Lokomat training on impaired ankle voluntary movement, known to be an important limiting factor in gait for iSCl patients.

Methods: Fifteen chronic iSCl subjects performed twelve 1-hour sessions of Lokomat training over the course of a month. The voluntary movement was qualified by measuring active range of motion, maximal velocity peak and trajectory smoothness for the spastic ankle during a movement from full plantar-flexion (PF) to full dorsi-flexion (DF) at the patient's maximum speed. Dorsi- and plantar-flexor muscle strength was quantified by isometric maximal voluntary contraction (MVC). Clinical assessments were also performed using the Timed Up and Go (TUG), the 10-meter walk (10MWT) and the 6-minute walk (6MWT) tests. All evaluations were performed both before and after the training and were compared to a control group of fifteen iSCl patients.
\end{abstract}

Results: After the Lokomat training, the active range of motion, the maximal velocity, and the movement smoothness were significantly improved in the voluntary movement. Patients also exhibited an improvement in the MVC for their ankle dorsi- and plantar-flexor muscles. In terms of functional activity, we observed an enhancement in the mobility (TUG) and the over-ground gait velocity (10MWT) with training. Correlation tests indicated a significant relationship between ankle voluntary movement performance and the walking clinical assessments.

Conclusions: The improvements of the kinematic and kinetic parameters of the ankle voluntary movement, and their correlation with the functional assessments, support the therapeutic effect of robotic-assisted locomotor training on motor impairment in chronic isCl.

\section{Background}

Individuals suffering from neurological diseases such as spinal cord injury (SCI) reveal several sensorimotor impairments. Previous studies report loss of voluntary control [1], muscle weakness, i.e., an inability to generate normal levels of muscle force [2,3], abnormal increase in muscle tone, i.e., spasticity $[2,4]$, and abnormal co-activation in muscular patterns $[5,6]$. A majority of patients with

\footnotetext{
* Correspondence: mehdi@northwestern.edu
'Sensory Motor Performance Program, Rehabilitation Institute of Chicago,

*Correspondence: mehdi@northwestern.edu
'Sensory Motor Performance Program, Rehabilitation Institute of Chicago, Chicago, USA

${ }^{2}$ Department of Physical Medicine and Rehabilitation, Northwestern University, Chicago, USA
}

incomplete motor SCI (iSCI) recovers a certain level of walking capacity but presents severe limitations [7]. Gait in iSCI is characterized by a number of features, including reduced speed, abnormal kinematic patterns and disrupted muscle activity [8]. To improve overground walking ability, locomotor therapies that combine a body-weight support (BWS) system with a treadmill have been developed over the past two decades $[9,10]$. BWS training is sometimes coupled with manual assistance from physical therapists $[11,12]$, functional electrical stimulation $[13,14]$, or most recently with robotic assistance $[15,16]$. Currently, there is no consensus regarding the most beneficial therapy to enhance ambulation in iSCI 
patients [17,18]. Robotic-assisted locomotor present several advantages, including the ability to increase the intensity and the total duration of training while maintaining a physiological gait pattern. Also, the task-specific practice of stepping is known to enhance the afferent feedback associated with normal locomotion and can induce plasticity in the involved motor centers [19-22]. Moreover, locomotor robotic devices can reduce personnel costs involved in manual assistance training (which can require up to three physical therapists) and can prevent excessive fatigue induced by electrical stimulation.

The Lokomat ${ }^{ø}$ (Hocoma AG, Volketswill, Switzerland), a driven-gait orthosis (DGO) device attached to a treadmill with BWS, was developed for locomotor therapy for various neurological diseases [23]. The device is composed of a motorized exoskeleton with bilateral electric drives that deliver a guidance force to the hip and knee joints, providing assistance during the swing and stance phases of the gait cycle. Previous research has shown that Lokomat training helps iSCI patients to improve their over-ground speed and gait endurance [15,16,24,25]. However, the mechanisms underlying these improvements are still unclear. The effectiveness of the robotic-assisted locomotor therapies is mostly quantified using clinical gait outcome measures (e.g., walking speed and functional independence); too few studies have investigated its specific effects on structures and functions involved in locomotion (e.g., neuroplasticity, muscle strength, gait pattern, etc.) [22,26-28]. However, recent work focused on understanding the mechanisms by which Lokomat training improves walking recovery capabilities seem promising. For example, it appears that training using the robotic device increases lower limbs' muscular activity [28,29] or cerebellum activation [22]. Moreover, Hidler et al. [26] demonstrated that, in a healthy population, the range of motion at the ankle joint is larger inside the Lokomat than on a treadmill. A larger range of motion could be helpful to improve the foot clearance during the swing phase and reduce the foot drop syndrome in iSCI.

The foot drop syndrome is considered as a strong limiting factor in the restoration of locomotion for those with neurological disease $[8,30]$. It is associated with a poor quality of locomotion, reduced functional capacity [31], and an enhanced risk of falling [32]. Researchers have observed deficient propulsion at the end of the stance phase, i.e., low plantar-flexion (PF) moment [33,34], and foot drag during the swing phase, i.e., reduced dorsiflexion (DF) movement [33,35]. Dorsi-flexors- particularly the tibialis anterior (TA) - play an important role in controlling the foot trajectory during the swing phase to ensure adequate foot clearance phase and to prevent foot drag. TA weakness can lead to reduce range of motion and reduced velocity peak during voluntary DF [36,37]. In iSCI patients, muscle weakness results from the combination of a deficit in cortico-spinal voluntary activation [38,39], muscular atrophy $[40,41]$ and increased stiffness in antagonist muscles $[42,43]$. Thus, locomotor training using the Lokomat seems promising to reduce these deficits and improve ankle DF movement.

This study focused on changes in ankle voluntary motor control in iSCI patients, considered as a strong indicator of recovery [44], after a robotic-assisted locomotor therapy. The aim was to quantify the effects of a 4-week Lokomat training regimen on the impaired voluntary movement of the ankle by measuring kinetic and kinematic parameters during a DF movement, and on patients' walking capacities assessed with popular clinical scales.

\section{Method}

\section{Participants}

Thirty ambulatory chronic SCI subjects with incomplete motor function loss due to trauma were recruited to participate in this study and randomly assigned to the two study groups. Half of the participants performed a 1-month Lokomat training (i.e., Intervention group) and the other half was assigned to the Control group. Groups were matched by age, time since injury, muscle tone at the ankle plantar-flexors assessed with the 6-point ordinal Modified Ashworth Scale (MAS) [45,46], and walking ability measured with the Walking Index for Spinal Cord Injury II (WISCI II). WISCI II assesses the patient's need for assistance or helping aids while walking over a short distance, using a scale ranging from 0 (patient unable to stand and/or participate in assisted walking) to 20 (patient ambulates with no device or physical assistance) $[47,48]$. Demographic characteristics for both groups are presented in Table 1.

All the participants met the following inclusion criteria: (a) motor-incomplete SCI with an American Spinal Injury Association impairment scale classification of $\mathrm{C}$ or D [49], (b) level of injury above T10, (c) ambulatory (i.e., showing the ability to take at least one step independently), (d) passive range of motion of the leg joints within functional limits for ambulation, and (e) medical clearance to participate. Patients from both groups were instructed to maintain a constant level of activity over the duration of the experiment. Subjects were draw from the outpatient service of the Rehabilitation Institute of Chicago. All of them provided their informed consent and the Northwestern University Institutional Review Board approved the study.

\section{Locomotor training}

Participants from the Intervention group participated in robot-assisted locomotor training using the Lokomat three times a week over four weeks, for a total of twelve training sessions. Each session lasted one hour, including 
Table 1 Baseline demographic characteristics and clinical scores of the Lokomat and Control groups

\begin{tabular}{|c|c|c|c|c|}
\hline & \multicolumn{2}{|c|}{ Lokomat group $(n=15)$} & \multicolumn{2}{|c|}{ Control group $(n=15)$} \\
\hline & Mean \pm S.D. & Range & Mean \pm S.D. & Range \\
\hline Age (y) & $50.80 \pm 2.12$ & $37-70$ & $44.65 \pm 2.66$ & $25-62$ \\
\hline Lesion duration $(\mathbf{y})$ & $11.80 \pm 2.54$ & $2-36$ & $8.09 \pm 1.89$ & $1-27$ \\
\hline WISCI II (range, 0-20) & $15.33 \pm 1.12$ & $9-20$ & $14.20 \pm 1.47$ & $9-20$ \\
\hline TUG (s) & $34.2 \pm 9.6$ & $9.7-126.1$ & $39.7 \pm 6.76$ & $8.7-95.0$ \\
\hline 10MWT $(\mathrm{m} / \mathrm{s})$ & $0.56 \pm 0.09$ & $0.05-1.06$ & $0.56 \pm 0.11$ & $0.09-1.49$ \\
\hline $6 \mathrm{MWT}(\mathrm{m})$ & $207.0 \pm 29.6$ & $25.5-346.0$ & $205.6 \pm 35.3$ & $25.9-501.2$ \\
\hline $\operatorname{Sex}^{\dagger}(F-M)$ & $7(46.67 \%)-8(53.33 \%)$ & $1(6.67 \%)-14(93.33 \%)$ & & \\
\hline Level of lesion ${ }^{\dagger}$ (C [C2-C7] - T [T1-T7]) & $11(60)-4(40)$ & $9(73.33)-6(26.67)$ & & \\
\hline Ankle flexors $\mathrm{MAS}^{\ddagger}$ (range, 0-4) & $2(\mathrm{IQR}=1)$ & $2(\mathrm{IQR}=1)$ & & \\
\hline
\end{tabular}

Continuous data are expressed as Mean \pm Standard Deviation [range], with the exception of categorical variables $\left({ }^{\dagger}\right)$ expressed as a number (\%) and nominal variables $\left({ }^{\ddagger}\right)$ expressed as the median (InterQuartile Range). Abbreviations: F, Female; M, Male; C, Cervical; T, Thoracic; MAS, Modified Ashworth Scale; WISCI II, Walking Index for Spinal Cord Injury II; TUG, Timed Up and Go; 10MWT, 10-meter walk; 6MWT, 6-minute walk.

set-up time, with between 30 and 45 minutes of training. The treadmill speed was increased from $1.5 \mathrm{~km} / \mathrm{h}$ to $3.0 \mathrm{~km} / \mathrm{h}$, as determined by patient tolerance and comfort, over the course of training. The guidance force, i.e., the level of control of the hip and knee joint trajectories in the sagittal plane provided by DGO, was progressively reduced during the training (from full to $20 \%$ assistance), as tolerated by the patient. Participants were instructed to "walk with the robot" to ensure lower-extremity movements that were consistent with the Lokomat stepping pattern. During the training, reductions in guidance force were prioritized over increasing walking speed to minimize the passive locomotor training and promote maximal voluntary muscle activation. The body-weight support was configured (from $95 \%$ to $25 \%$ body weight) to maximize lower-extremity loading without producing excessive knee flexion during the stance phase or allowing to toes to drag during the swing phase. The three training parameters (i.e., walking speed, guidance force and body-weight support) were adjusted to guarantee the highest amount of active subject participation and to provide a challenge to them over the training's sessions. Straps secured the heads of the metatarsals to the foot supports on the robot to ensure a neutral position of the ankle (i.e., ankle angle of $90^{\circ}$ ). Patients were asked to pay attention to their ankle movements during the gait cycle. We encouraged patients to lift their toes during the swing phase and focus on planting the heel of the forward foot on the treadmill first. A mirror was placed before the patient to allow him to monitor his leg movements during the training.

\section{Clinical assessments}

Patients performed three clinical evaluations to assess their functional ambulation capacity, including the Timed Up and Go test (TUG), the 10-meter walk test (10MWT), and the 6-minute walk test (6MWT). TUG evaluates the mobility and functional ambulation by measuring time taken by the subject to stand up from an armed chair, walk for 3 meters, turn around, return, and sit down into the chair [50]. 10MWT is used to evaluate preferred walking speed by measuring the time spent to walk a distance of 10 meters [51].

$6 \mathrm{MWT}$ is used to assess walking endurance capacity by measuring the distance in meters covered by $6 \mathrm{mi}$ nutes of walking [31]. Clinical evaluations were performed at two time intervals: before (baseline) and after the complete Lokomat training period (one month) for the Intervention group; for the Control group, baseline and 1-month evaluations were also performed. Baseline scores for both groups are presented in Table 1.

\section{Ankle kinematic and kinetic assessments Apparatus and recording}

The voluntary movement capacity and muscle strength of the ankle were evaluated at the same two time intervals as the clinical assessments (i.e., baseline and 1month). For this purpose, participants were seated in an experimental chair with the thigh strapped to the chair base (Figure 1). The ankle was secured to a rigid "footrest" which was in turn attached to the rotational axis of a servomotor, allowing for DF and PF of the ankle. The seat and footrest were adjusted such that the ankle and knee joints were flexed at $90^{\circ}$ (ankle) and $130^{\circ}$ (knee). A rotary encoder recorded angular position, while a 6 -axis torque transducer recorded torque; this transducer was aligned such that the torque was measured about the ankle axis of rotation. Position and torque data were sampled at $1 \mathrm{kHz}$ by a 16 bit A/D converter, and antialias filtered on-line at $200 \mathrm{~Hz}$. A $90^{\circ}$ angle between the calf and foot was considered as the neutral position for the ankle and defined as zero degrees; DF was considered positive by convention. 


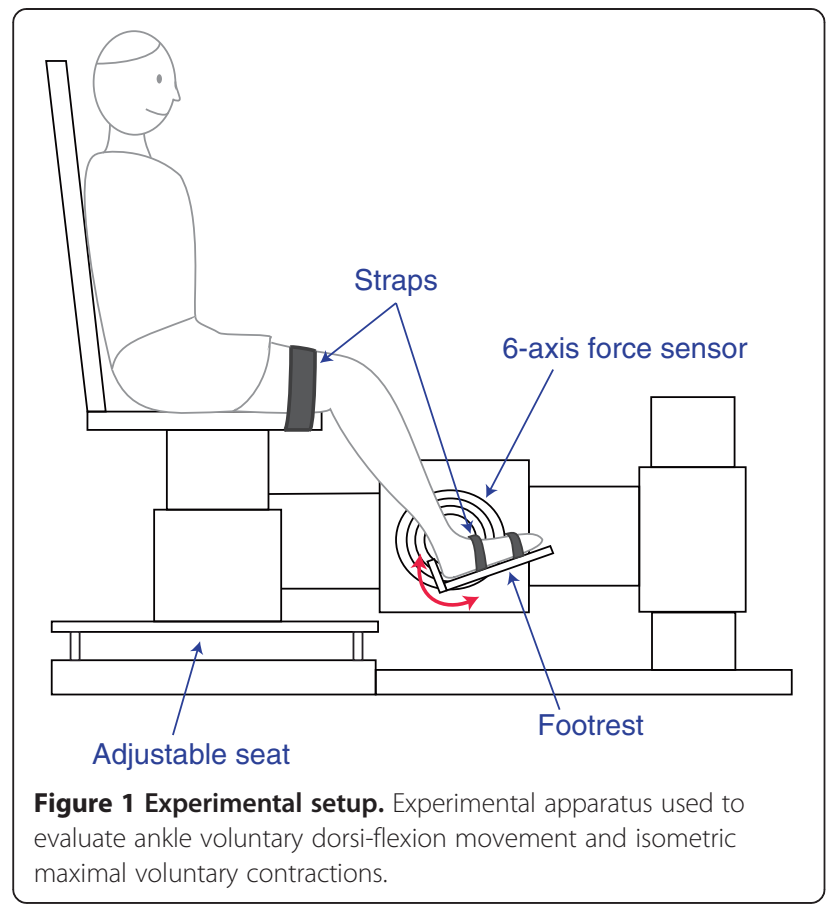

\section{Experimental procedure}

To evaluate DF voluntary movement capacity, subjects were instructed to rotate their ankle from full PF position to full DF position at their maximum possible speed. The initial full PF position was defined as the maximal position voluntarily reached by the subject in the PF direction without assistance. We repeated this task twice.

Prior to the voluntary task, the passive range of motion (PROM) was determined by an examiner manually stretching the patient's ankle attached to the footrest in both directions, at a very slow (quasi-static) speed as to prevent muscle activation, until maximal resistance or pain was encountered.

We also measured the isometric maximal voluntary contraction (MVC) of the ankle plantar- and dorsiflexors while the ankle was held fixed at the neutral position. Participants were instructed to voluntarily contract their plantar-flexors by pushing down with their toes onto the footrest with as much effort as possible; to contract dorsi-flexors, patients were instructed to lift their toes up. Subjects were encouraged to sustain the MVC contraction for 5 seconds in each direction.

While SCI typically affects both sides of the body, in this study only the ankle with the lowest isometric maximum voluntary torque in the DF direction and the highest MAS score was examined.

\section{Data reduction}

Angular velocity and acceleration were computed as the first and second derivatives of the ankle angular position (Figure 2). The onset of movement was defined as the

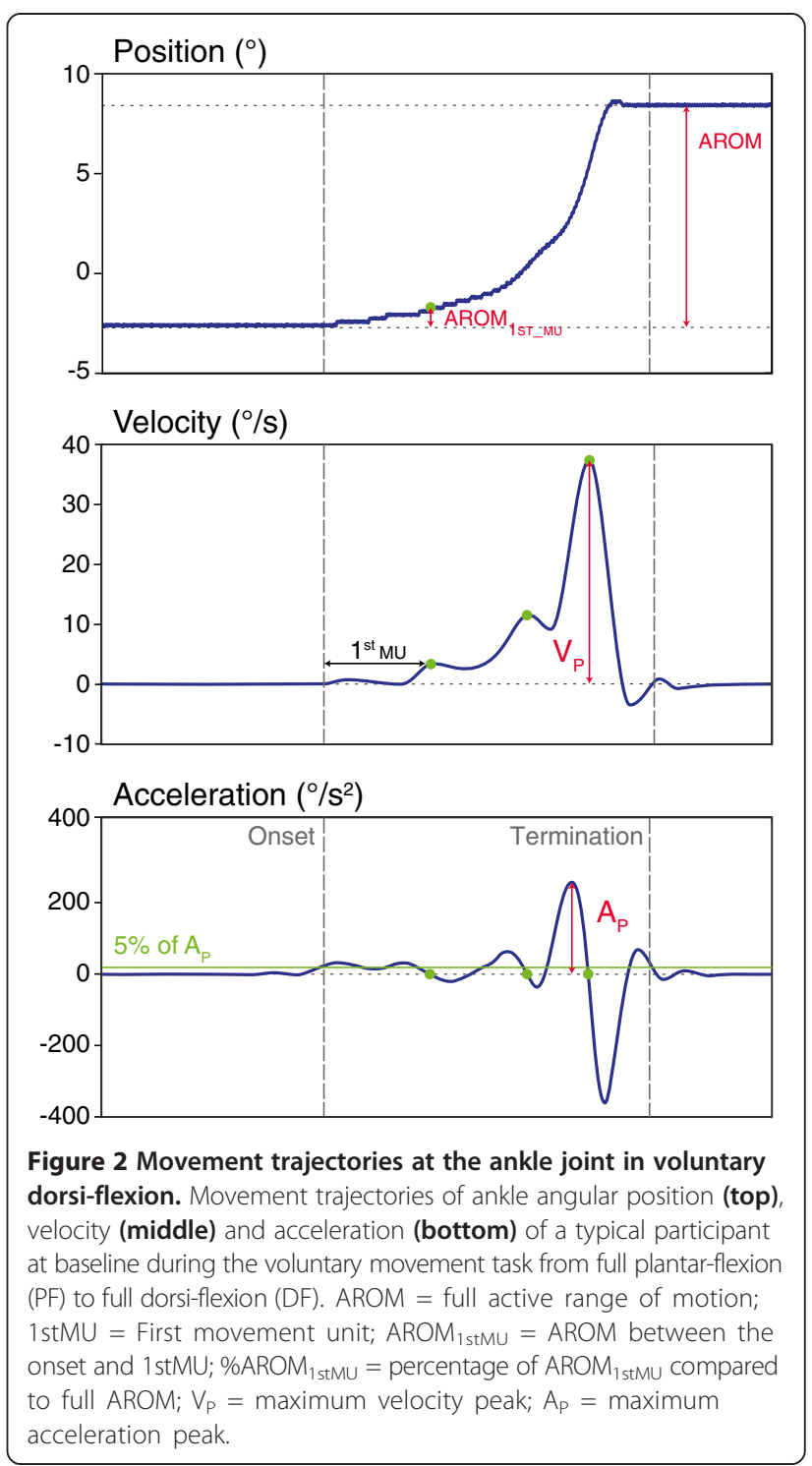

first sample whose acceleration was greater than 5\% of the maximum acceleration peak; the termination of movement was defined once the acceleration dropped below this value. The first movement unit (1stMU) was defined as the first peak (local maximum) in velocity after the movement onset. The times of peak velocity were detected in the acceleration profile as the time points where the acceleration changed sign from positive (acceleration) to negative (deceleration) through zero.

From this data, the full active range of motion (AROM) at the ankle joint was computed between the onset and the termination of movement, as well as the maximum angular velocity peak $\left(\mathrm{V}_{\mathrm{P}}\right)$. Smoothness of the movement was estimated using two variables: (i) $\mathrm{AROM}_{1 \mathrm{stMU}}$, the AROM between the onset and the first movement unit, and (ii) $\% \mathrm{AROM}_{1 \mathrm{stMU}}$, the percentage of $\mathrm{AROM}_{1 \mathrm{stMU}}$ compared to the full AROM. Our earlier study suggests 
that these two variables are sensitive enough to detect changes in movement smoothness in SCI patients [52]. These kinematic measures indicate the ability of the patient to scale muscle force to accomplish the movement in a single accelerating and decelerating cycle [52].

Maximum isometric torque was computed during the 5-second MVC hold in each direction (DF and PF). The average torque during the 3 -second window in which the torque exhibited the smallest standard deviation was used as the MVC torque; the largest magnitude plantarflexion $\left(\mathrm{MVC}_{\mathrm{PF}}\right)$ and dorsi-flexion $\left(\mathrm{MVC}_{\mathrm{DF}}\right)$ torque of the two trials was used.

\section{Statistical analysis}

Since Kolmogorov-Smirnov tests of normality indicated a skewed distribution of data, non-parametric tests were used for all the following analysis.

To test the homogeneity between Control and Intervention groups at the inclusion, Wilcoxon signed-rank tests were conducted on the continuous variables (i.e., Age, Lesion duration and WISCI II) and Chi-squared tests were performed on the categorical and ordinal variables (i.e., Level of lesion and MAS).

In order to determine the effects of the Lokomat training on the ankle voluntary movement and walking capacities, we compared the MVC values, the kinematic parameters for the DF task, and the clinical evaluations of walking capacity assessed at baseline and one month. Wilcoxon signed-rank tests were performed on baseline and 1-month data independently for each group for the following parameters: PROM, AROM, $V_{\mathrm{B}} \mathrm{AROM}_{1 \mathrm{stMU}}$, $\% \mathrm{AROM}_{1 \mathrm{stMU}}, \mathrm{MVC}_{\mathrm{PF}}, \mathrm{MVC}_{\mathrm{DF}}, \mathrm{TUG}, 10 \mathrm{MWT}$ and 6MWT.

We used Spearman rank correlation analysis to determine the relationships between the three clinical assessments (i.e., TUG, 10MWT and 6MWT) and the ankle's kinematic and kinetic parameters at baseline (i.e., AROM, $\mathrm{V}_{\mathrm{P}}, \mathrm{AROM}_{1 \mathrm{stMU}}, \% \mathrm{AROM}_{1 \mathrm{stMU}}, \mathrm{MVC}_{\mathrm{PF}}$ and $\left.\mathrm{MVC}_{\mathrm{DF}}\right)$.

The significance level was set at $\mathrm{P}=0.05$.

\section{Results}

\section{Relationship between walking assessments and ankle} ability measures

We investigated the relationship between ankle voluntary movement capabilities (quantified by our kinematic and kinetic variables) and walking capacities (assessed by clinical scales) using correlation analysis on baseline measurements. The correlation coefficients are presented in Table 2. Both the mobility assessed with the TUG and the over-ground walking speed measured with the 10MWT showed significant correlations with AROM, AROM $\mathrm{AstMU}_{\text {, }}$ $\mathrm{MVC}_{\mathrm{PF}}$ and $\mathrm{MVC}_{\mathrm{DF}}$ (All Ps <0.05). Thus, these two components of ambulation are related to ankle ability, as measured by active range of motion, movement smoothness and muscle strength. Moreover, the $6 \mathrm{MWT}$ assessing the endurance capacity was significantly correlated with AROM, $V_{\mathrm{P}}, \mathrm{AROM}_{1 \mathrm{stMU}}$ and $\mathrm{MVC}_{\mathrm{DF}}$ (All Ps < 0.05). To correct for these multiple tests, we have used Bonferroni correction to adjust the alpha level for all our correlation analysis. Since we made 18 tests, a level of significance was set at $0.0028(0.05 / 18)$ for which all correlations reported significant (Table 2) remained so except one related to the relationship between TUG and $\mathrm{MVC}_{\mathrm{PF}}$.

To summarize, correlation tests show that the variables used to describe the kinematic components of the DF voluntary movement and the muscle strength at the ankle joint were related to popular measures of mobility and walking capacities used in the clinic.

Therapeutic effects of robotic-assisted locomotor training At the first evaluation (baseline), the two groups were homogeneous. No statistically significant differences were found in terms of age $(P=0.09)$, level of lesion $(\chi 2=0.6$, $\mathrm{df}=1, \mathrm{P}=0.44)$, duration of lesion $(\mathrm{P}=0.46)$, walking capacity measured with WISCI II $(\mathrm{P}=0.61)$ and plantarflexor muscle tone assessed with MAS $(\chi 2=5.12$, $\mathrm{df}=4$, $\mathrm{P}=0.27)$.

\section{Ankle passive range of motion and muscle tone}

For the Intervention group, a significant improvement in the ankle PROM was observed over time $(\mathrm{P}<0.05)$. The mean \pm standard error of PROM was $62.33^{\circ} \pm 2.06^{\circ}$ at baseline and $64.33^{\circ} \pm 2.23^{\circ}$ at one month. For the Control group, PROM was similar between the two evaluations (baseline: $58.00^{\circ} \pm 2.62^{\circ}$; 1 -month: $57.33^{\circ} \pm 2.92^{\circ}$; $\mathrm{P}=0.75)$.

Regarding plantar-flexor muscle tone assessed with MAS, no change is observed in the 1-month interval for both groups (Intervention group: $\chi 2=5.78, \mathrm{df}=4, \mathrm{P}=0.22$; Control group: $\chi^{2}=4.50, \mathrm{df}=4, \mathrm{P}=0.21$ ).

\section{Ankle dorsiflexion voluntary movement: kinematic measures}

For the Intervention group, the analysis showed that three of the four kinematic parameters that were used to quantify the voluntary DF performance presented an improvement over the course of the Lokomat training. Paired-comparisons revealed a significantly greater AROM $(\mathrm{P}<0.05), \mathrm{V}_{\mathrm{P}}(\mathrm{P}<0.05)$ and $\mathrm{AROM}_{1 \mathrm{stMU}}(\mathrm{P}<0.05)$ at one month compared to baseline (Figure 3). For AROM, subjects exhibited an average increase of $27.5 \pm 9.9 \%$ (5.13 \pm $1.63^{\circ}$ ), improving from $28.80 \pm 3.84^{\circ}$ to $33.93 \pm 3.89^{\circ}$. For $\mathrm{V}_{\mathrm{B}}$ we observed an average increase of $27.54 \pm 12.24 \%$ $(15.90 \pm 7.16 \%$ s); at baseline the average maximum velocity peak was $93.97 \pm 13.49^{\circ} / \mathrm{s}$, while it had increased to $109.87 \pm$ $14.54^{\circ} \mathrm{s}$ after one month. For $\mathrm{AROM}_{1 \mathrm{stMU}}$, the average increase is of $38.88 \pm 17.66 \%\left(2.79 \pm 0.95^{\circ}\right)$ from $11.48 \pm$ $1.50^{\circ}$ to $14.27 \pm 1.79^{\circ}$. However, there is no significant 
Table 2 Correlations ( $r$ values) between the walking clinical assessments and the ankle voluntary movement parameters at baseline

\begin{tabular}{|c|c|c|c|c|c|c|}
\hline & \multicolumn{4}{|c|}{ Kinematic } & \multicolumn{2}{|l|}{ Kinetic } \\
\hline & AROM & $V_{P}$ & AROM $_{1 \mathrm{stMU}}$ & 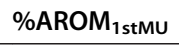 & $\mathrm{MVC}_{\mathrm{PF}}$ & $\mathrm{MVC}_{\mathrm{DF}}$ \\
\hline TUG & $-0.57^{*}$ & -0.37 & $-0.47^{*}$ & 0.22 & $-0.41^{*}$ & $-0.52^{*}$ \\
\hline 10MWT & $-0.53^{*}$ & -0.34 & $-0.48^{*}$ & -0.11 & $-0.44^{*}$ & $-0.50^{*}$ \\
\hline $6 \mathrm{MWT}$ & $0.70^{*}$ & $0.73^{*}$ & $0.74^{*}$ & 0.02 & 0.36 & $0.63^{*}$ \\
\hline
\end{tabular}

Abbreviations: TUG, Timed Up and Go; 10MWT, 10-meter walk test; $6 M W T$, 6-minute walk test, $A R O M$, Active Range of motion; $V_{P}$, Velocity peak. AROM 1 stMU, AROM between the onset and the first movement unit; \%AROM ${ }_{15 t M U}$, Percentage of $A_{R O M}{ }_{15 t M U}$ compared to full AROM; $M V C_{P F}$ and $M V C_{D F}$, Maximal voluntary contraction in dorsi- and plantar-flexion directions. *represents significant correlations $(P<0.05)$.
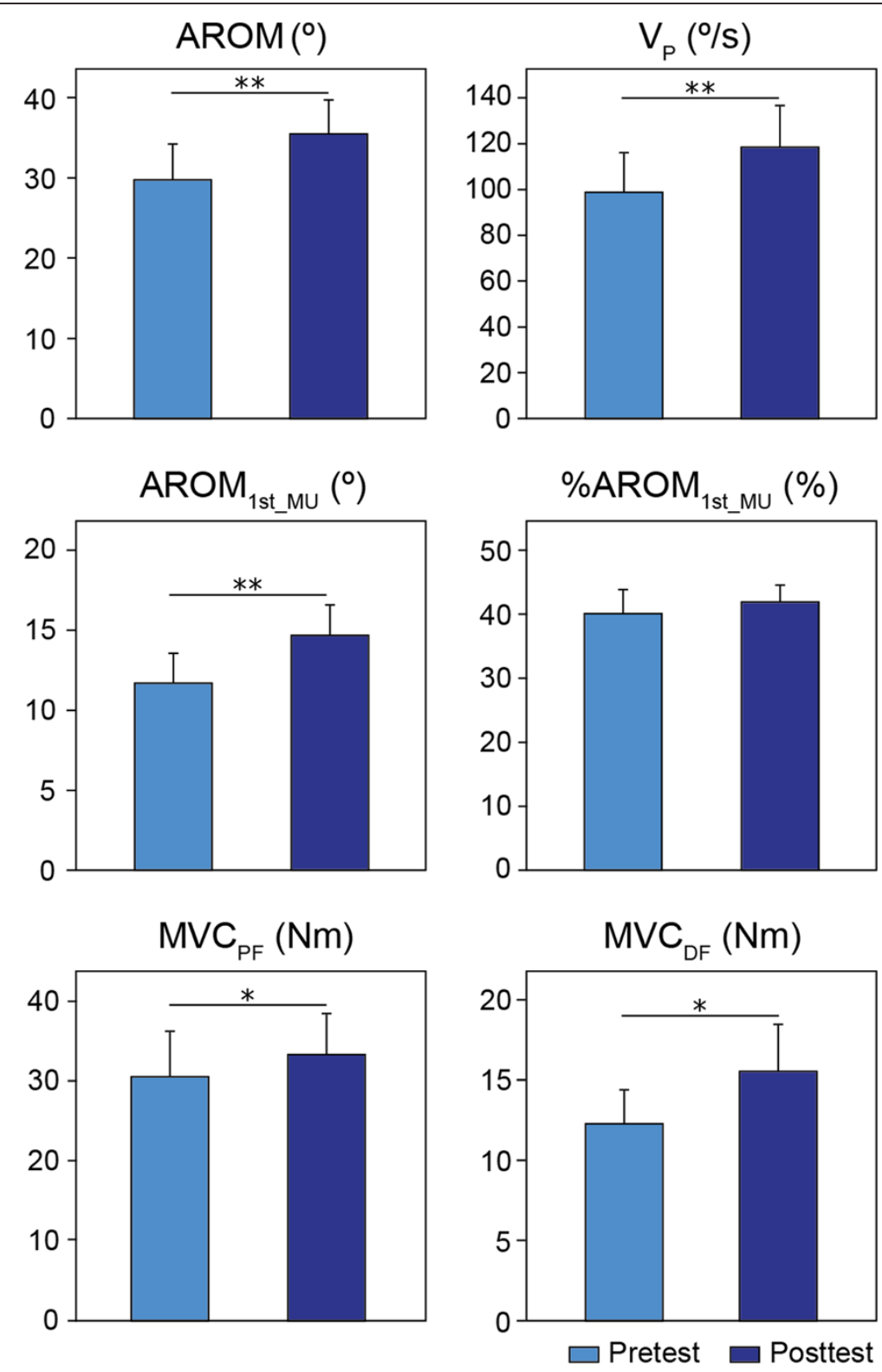

Figure 3 Baseline and 1-month average in the ankle voluntary movement for the Intervention group. Kinematic (AROM and $V_{p}$ ), smoothness $\left(A R O M_{1 s t M U}\right.$ and \%AROM $\left.{ }_{1 s t M U}\right)$ and kinetic ( $M V C_{P F}$ and $M V C_{D F}$ ) group averages before and after one month of Lokomat training. Bars represent the standard error of the group mean. Asterisks indicate a statistical comparison between pre- and posttests, where *indicates significance at $\mathrm{P}<0.05$; **indicates significance at $\mathrm{P}<0.01$. 
change over time for $\% \mathrm{AROM}_{1 \mathrm{stMU}}(\mathrm{P}=0.64)$. That is, patients from the Intervention group were able to voluntarily move their ankles farther and faster after training. In general, these participants exhibited an improvement in the kinematic and smoothness components of their movement during the voluntary DF task.

In contrast, for the Control group, no statistically significant difference was found between the two tests in AROM $(\mathrm{P}=0.95), \mathrm{V}_{\mathrm{P}}(\mathrm{P}=0.34), \mathrm{AROM}_{1 \mathrm{stMU}}(\mathrm{P}=0.59)$ and $\% \mathrm{AROM}_{1 \mathrm{stMu}}(\mathrm{P}=0.50)$.

\section{Ankle maximum isometric voluntary contractions}

We quantified the changes in plantar- and dorsi-flexor muscle strength at the ankle joint over the training, using baseline vs. 1-month comparisons of the mean MVC torque. For the Intervention group (Figure 3), an average increase of $14.79 \pm 4.71 \%$ was observed in the PF direction $(\mathrm{P}<0.05)$, with the mean torque increasing from $26.77 \pm 4.42 \mathrm{Nm}$ at baseline to $29.13 \pm 4.10 \mathrm{Nm}$ at one month. In the DF direction, $\mathrm{MVC}_{\mathrm{DF}}$ improved significantly by $24.04 \pm 7.91 \%$ at one month compared to baseline $(\mathrm{P}<0.05)$, from a baseline mean of $10.95 \pm$ $1.67 \mathrm{Nm}$ to a one month mean of $13.55 \pm 2.31 \mathrm{Nm}$. The relatively larger variance after training, compared to baseline, in the DF direction suggests high variability in individual responses to the Lokomat training as illustrated in Figure 4. We observed two different recovery trends: nine of our fifteen participants $(63 \%$ of the subjects) presented an increase in $\mathrm{MVC}_{\mathrm{DF}}$ of $10-106 \%$

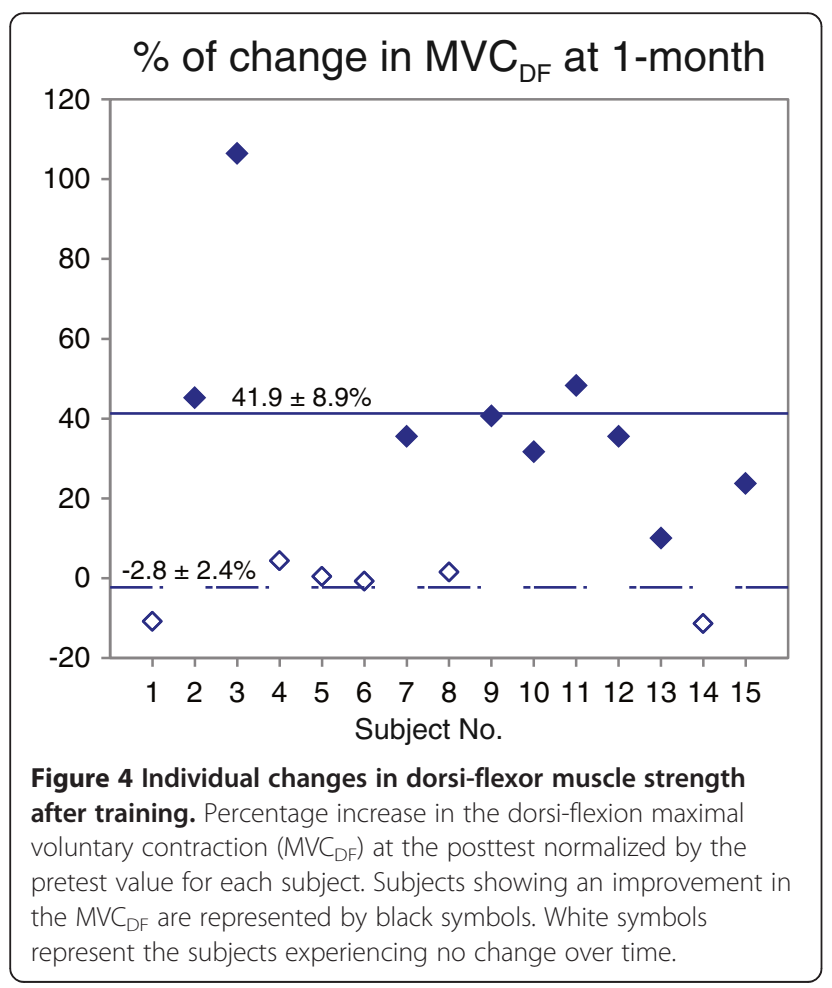

(average change: $41.9 \pm 8.9 \%$ ), while the rest of the group experiences no change over time (average change: $-2.8 \pm$ $2.4 \%)$.

In summary, results of MVC tests show an improvement in the strength of dorsi- and plantar-flexor muscles during isometric voluntary contraction after the 1month Lokomat training. On the other hand, for the Control group, no change was observed between the two tests in $\mathrm{MVC}_{\mathrm{PF}}(\mathrm{P}=0.09)$ and $\mathrm{MVC}_{\mathrm{DF}}(\mathrm{P}=0.81)$.

\section{Walking performance}

To evaluate the effectiveness of the Lokomat training in terms of functional capabilities, we compared the performance of the Intervention group for three clinical measures of ambulation impairment (i.e., TUG, 10MWT and 6MWT) before and after the training. The TUG showed a significant reduction of $13.99 \pm 3.53 \%$ in the time needed to perform the task $(\mathrm{P}<0.05)$. The mean time to perform the task was $34.15 \pm 9.61 \mathrm{~s}$ before training and $27.83 \pm 7.32 \mathrm{~s}$ after the Lokomat training. The 10MWT exhibited a significant increase in the mean over-ground gait speed $(0.08 \pm 0.02 \mathrm{~m} / \mathrm{s})$ after training $(\mathrm{P}<0.05)$, from a mean speed of $0.56 \pm 0.09 \mathrm{~m} / \mathrm{s}$ pretraining to a mean speed of $0.64 \pm 0.10 \mathrm{~m} / \mathrm{s}$ post-training. This change corresponded to an improvement of $13.4 \pm$ $2.8 \%$. These results demonstrate an improvement in mobility (TUG) and walking speed (10MWT) after one month of robotic-assisted locomotor practice. However, no significant change in the patients' endurance capacity was observed between the two tests, as assessed by the $6 \mathrm{MWT}(\mathrm{P}=0.46)$ : the mean walking distance was $206.96 \pm 29.57 \mathrm{~m}$ before training and $208.87 \pm 28.36 \mathrm{~m}$ after the training.

For the Control group, no statistically differences were found between the two tests in TUG (P = 0.45), 10MWT $(\mathrm{P}=0.36)$ and 6MWT $(\mathrm{P}=0.17)$.

\section{Discussion}

The present study is, to our knowledge, the first to have investigated the effects of a one-month Lokomat training program on the voluntary movement of the ankle joint in chronic iSCI patients. Our results demonstrated that a benefit of this locomotor training could be to improve ankle voluntary DF movement in terms of range of motion, velocity and smoothness. Moreover, a gain in the strength of the dorsi- and plantar-flexor muscles was seen after the Lokomat training. The clinical gait assessments performed indicated that the Lokomat training could drive an improvement in mobility and over-ground walking velocity. In addition, correlations observed between our kinematic and kinetic variables and these clinical measures suggest that the parameters examined in this study may help to better understand the mechanisms which underlie improvements 
in walking capacity following robotic-assisted locomotor training.

\section{Lokomat training effects on ankle muscle strength}

To our knowledge, this is the first time that the effect of Lokomat training on the strength of the ankle plantarflexor muscles was directly investigated. The improvement in plantar-flexor strength is a key element in gait recovery as the forward progression during locomotion is primarily produced by this muscle group [53,54]. Our results show that the maximal torque generated during the $M V C_{P F}$ task increases significantly from the baseline after the Lokomat training. This finding confirms earlier observations that repetitive locomotor training in chronic iSCI patients is beneficial to developing strength in the PF muscle group [55]. This improvement could be the expression of the muscle plasticity phenomenon resulting from the extra loading on the lower limb [56,57], as well as an enhancement of voluntary activation drive by the intensive repetition of an "unused" task [55,58].

Weakness in the dorsi-flexor muscles is one of the major causes of impairment in foot clearance during the swing phase (i.e., foot drop syndrome) [30]. Thus, recovery of the strength of ankle dorsi-flexor muscles is essential to prevent excessive plantar-flexion movement. Our results indicate an increase in $\mathrm{MVC}_{\mathrm{DF}}$ generated after the training. Only one earlier study reports an augmentation of the tibialis anterior muscle activity in a Lokomat training when resistance is added during the swing phase [29]. Similar to plantar-flexor muscles, the increase in $M V C_{D F}$ level could find its origin in the improvement of voluntary activation of ankle dorsi-flexor muscles $[38,59]$. Previously, an enhancement in the cortico-spinal tract function has been observed for the tibialis anterior following treadmill training in chronic SCI patients [60]. In our point of view, the unique configuration provided by the Lokomat can be beneficial to improve ankle voluntary activation. By actively guiding hip and knee joints through DGO in a physiological stepping pattern, a patient can focus his attention on his ankle movements during the gait cycle. Moreover, the strap placed around footwear maintaining the foot in a neutral position allows the dorsiflexor muscles contract to their shortened length, a condition known to promote the best recovery of function as suggested by Ada et al. [61].

A diminution of spasticity in the antagonist (i.e., plantarflexor) muscles would reduce its reciprocal inhibitory effects on the dorsi-flexors muscles, particularly the TA activation $[42,62,63]$, and could also explain the increase in $\mathrm{MVC}_{\mathrm{DF}}$. In our study, no significant difference has been observed in plantar-flexor muscle tone assessed with MAS between baseline and 1-month. However reliability and reproducibility of MAS is unclear [64], particularly regarding the assessment of spasticity in the lower extremities in the
SCI population [65-67]. A recent study showing a reduction of the reflex and intrinsic components of the ankle stiffness in chronic iSCI subjects undergoing Lokomat training offers some support to this hypothesis [68]. Also, the increase in $\mathrm{MVC}_{\mathrm{DF}}$ might be the expression of the enhancement of voluntary activity in the TA due to the reduction of the inhibitory dorsiflexor responses. On the other hand, the lack of improvement in some subjects could be the consequence of atrophic dorsi-flexor muscles, a common phenomenon in chronic SCI patients [40].

The different recovery patterns observed in $\mathrm{MVC}_{\mathrm{DF}}$ (i.e., an increase observed in half of our study participants and no change for the other half) should be investigated further to better understand the mechanisms underlying the recovery of dorsi-flexor muscle activity following Lokomat training.

\section{Ankle dorsi-flexion kinematic improvement}

Ankle dorsi-flexion restoration is fundamental in iSCI patients to prevent foot drag and improve walking capabilities [31]. The correlation tests performed in this study showed a relationship between the walking clinical assessments (i.e., TUG, 10MWT and 6MWT) and the variables used to quantify the ankle dorsi-flexion voluntary movement (i.e., AROM, $\mathrm{V}_{\mathrm{B}} \mathrm{AROM}_{1 \mathrm{stMU}}$ ). Patients who presented the highest walking capacities also showed the best performance for the ankle voluntary movement from full PF position to full DF position. Our results show that after four weeks of Lokomat training, subjects increase their range of motion, their maximum velocity, and the smoothness of their motion.

At the ankle joint, the AROM (measured from maximal PF to maximal DF) is limited by the interaction between (i) the force produced by the dorsi-flexor muscles, (ii) the passive resistance from the stretched structures and (iii) the spastic co-contraction in the antagonist muscles (i.e., plantar-flexors). As the increase in $\mathrm{MVC}_{\mathrm{DF}}$ was observed in nine of our fifteen patients, the gain observed in AROM for all our patients cannot be entirely due to an enhancement in dorsi-flexor muscle strength. On the other hand, the significant improvement in the passive range of motion found after the 1-month training suggests a reduction in the resistance of the passive tissues. During the Lokomat training, the support provided by foot straps can prevent both an excessively shortened position of the plantar-flexor muscles as well as incapacitating contractures. Thus, this "stretching" of calf muscles could have a beneficial effect on the passive ankle joint stiffness [69] and by extension improve the ankle DF voluntary movement. The reduction of the spastic co-contraction in the antagonist muscles observed after a Lokomat training in an earlier study would also serve to increase the ankle AROM [68]. In addition, a diminution in the spasticity in our subjects would 
explain the improvement in other parameters of the ankle DF voluntary movement (i.e., velocity peak and smoothness); previous studies have observed some degree of causality between spasticity and the velocity and smoothness of voluntary movement [70-73]. In fact, Latash and Penn [72] demonstrated an improvement in the peak speed and smoothness of ankle movement when muscle spasticity was reduced by anti-spastic medication.

\section{Walking capacity improvement}

In terms of functional capacities, the results obtained for the clinical assessments show an improvement of mobility (i.e., TUG) and over-ground walking speed (i.e., 10MWT) after one month of Lokomat training for our iSCI subject group. These observations are consistent with the literature on robotic-assisted gait devices showing a beneficial effect on the ambulation capacities $[15,16,18,22]$. The improvement in walking speed is particularly noteworthy; we observed an average increase of $0.08 \mathrm{~m} / \mathrm{s}$, which is greater than the minimally important difference of $0.05 \mathrm{~m} / \mathrm{s}$ reported by Musselman after a BWS treadmill training [73]. Two factors evaluated in this study might contribute to the observed gain in mobility and velocity: (i) improvements in the strength of the ankle plantar-flexor muscle group, which provides most of the energy required for forward progression [53,54], and (ii) enhancements to the dorsiflexion voluntary movement, which allows for adequate clearance during the swing phase [8].

The absence of changes in the 6MWT-which evaluates the endurance component of the gait capacity-might be explained by the duration of the training provided to our participants. In the study, we administered twelve 1-hour Lokomat training sessions over a month, but the endurance gain in over-ground gait has been observed for robotic-assisted locomotor training studies with training durations that are at least twice as long [15,44]. A longer and/or more intensive training regimen might be helpful to develop endurance capacity in the chronic iSCI population.

\section{Study limitations and implications}

Our study characterized the impaired voluntary movement at the ankle joint using a specific task that involved only the joint by itself, and only articulated the joint through PF and DF. The confirmation of our findings during a functional task as walking (e.g., an assessment of the spatio-temporal trajectories of the ankle joint during the gait cycle) would be helpful to confirm the clinical significance of the physical intervention (Lokomat training). Comparison of gait kinematics within this population can be complicated by differences in self-selected speed and gait pattern, and the presence of braces, orthotics, and gait assistance device (such as walkers). The present experimental design attempts to control for these differences by isolating only the DF/PF motion. Also, technical and methodological limitations prevent the assessment of many ankle kinematic and kinetic variables during standard gait. However, the relationships found between our variables and the clinical measures indicate that the ankle voluntary movement limitations are related to walking capacity, thus suggesting a therapeutic benefit of the Lokomat training.

The observation of individual trends in the recovery of dorsi-flexor strength illustrates that Lokomat training induces different patterns of recovery among patients, as expected from any therapeutic intervention. Thus, to fully characterize the effects of the robotic-assisted locomotor therapy, we need to further investigate the different recovery patterns identified in the recovery of dorsi-flexor muscle strength. A systematic assessment of the spasticity phenomenon at the ankle joint, as well as the identification of skeletal muscle atrophy and assessment of cortico-spinal function, would be helpful to determine the underlying mechanisms involved in the ankle voluntary movement recovery. A complete understanding of these mechanisms is crucial for identifying patients for whom a robotic-assisted locomotor therapy would be the most beneficial (i.e., to achieve the best functional outcome), as well as to specify an appropriate training duration and intensity which would maximize positive results.

Finally, researchers who performed the tests were not blinded for group allocation because they needed to stay with the subjects during the training and experimental protocol.

\section{Clinical significance}

Our current findings suggest that a 1-month Lokomat training regimen is beneficial to improve ankle voluntary movement in a chronic iSCI population. Even if the Lokomat training does not provide an active control of the ankle, its impact on this joint should not be neglected, as well as the instructions given to maximize the contribution of the ankle during the training. The correlation of these parameters with the walking measures is promising to assess the efficacy of robotic-driven locomotor training. The specific pathways by which ankle voluntary movement is enhanced remain to be investigated. However, an improvement of voluntary activation of dorsi-flexors muscles associated with a reduction of passive and neuromuscular components of joint stiffness might explain the changes observed following the 1-month training. The findings of this study help to improve the understanding of how the Lokomat training affects the recovery of walking capacities in patients with chronic iSCI.

\section{Competing interest}

The authors declare that they have no competing interests. 


\section{Authors' contributions}

DV and XN were involved in subject recruitment, data collection, and Lokomat training. DV performed the analysis and interpretation of data and wrote the manuscript. XN assisted in revising the manuscript. MMM designed the study, supervised data collection and analysis, and participated in interpreting and revising the manuscript. All authors read and approved the final manuscript.

\section{Acknowledgements}

This study was supported by the National Institutes of Health (NICHD- R01HD059895) and the Craig H. Neilsen Foundation awards to MMM. We thank Matthew Kindig for his editorial assistance and Petra Conaway for her help in the Lokomat training.

Received: 14 March 2014 Accepted: 24 March 2014

Published: 31 March 2014

\section{References}

1. Thomas CK, Zaidner EY, Calancie B, Broton JG, Bigland-Ritchie BR: Muscle weakness, paralysis and atrophy after human cervical spinal cord injury. Exp Neurol 1997, 148:414-423.

2. Rymer WZ, Power RK: Muscular weakness in incomplete spinal cord injury. Compr Ther 1987, 13:3-7.

3. Thomas CK, Tucker ME, Bigland-Ritchie BR: Voluntary muscle weakness and coactivation after chronic cervical spinal cord injury. J Neurotrauma 1998, 15:149-161.

4. Gerhart KA, Bergstrom E, Charlifue SW, Menter RR, Whiteneck GG: Longterm spinal cord injury: functional changes over time. Arch Phys Med Rehabil 1993, 74:1030-1034.

5. Dietz V, Colombo G, Jensen L: Locomotor activity in spinal man. Lancet 1994, 344:1260-1263.

6. Lim H, Lee D, McKay W, Priebe M, Holmes S, Sherwood A: Neurophysiological assessment of lower-limb voluntary control in incomplete spinal cord injury. Spinal Cord 2005, 43:283-290.

7. Waters RL, Adkins R, Yakura J, Vigil D: Prediction of ambulatory performance based on motor scores derived from standards of the American Spinal Injury Association. Arch Phys Med Rehabil 1994, 75:756-760.

8. Barbeau H, Pépin A, Norman KE, Ladouceur M, Leroux A: Review: walking after spinal cord injury: control and recovery. Neuroscientist 1998, 4:14-24.

9. Barbeau H, Blunt R: "A novel interactive locomotor approach using body weight support to retrain gait in spastic paretic subjects," Plasticity of Motoneuronal Connections. Amsterdam, the Netherlands: Elsevier; 1991:461-474.

10. Wernig A, Müller S: Laufband locomotion with body weight support improved walking in persons with severe spinal cord injuries. Spinal Cord 1992, 30:229-238.

11. Behrman AL, Harkema SJ: Locomotor training after human spinal cord injury: a series of case studies. Phys Ther 2000, 80:688-700.

12. Visintin M, Barbeau H, Korner-Bitensky N, Mayo N: A new approach to retrain gait in stroke patients through body weight support and treadmill stimulation. Stroke 1998, 29:1122-1128.

13. Ladouceur $M$, Barbeau H: Functional electrical stimulation-assisted walking for persons with incomplete spinal injuries: longitudinal changes in maximal overground walking speed. Scan J Rehabil Med 2000, 32:28-36.

14. Ladouceur $M$, Barbeau H: Functional electrical stimulation-assisted walking for persons with incomplete spinal injuries: changes in the kinematics and physiological cost of overground walking. Scan J Rehabil Med 2000 32:72-79.

15. Hornby TG, Zemon DH, Campbell D: Robotic-assisted, body-weightsupported treadmill training in individuals following motor incomplete spinal cord injury. Phys Ther 2005, 85:52-66.

16. Wirz M, Zemon DH, Rupp R, Scheel A, Colombo G, Dietz V, Hornby TG: Effectiveness of automated locomotor training in patients with chronic incomplete spinal cord injury: a multicenter trial. Arch Phys Med Rehabil 2005, 86:672-680.

17. Nooijen CF, ter Hoeve N, Field-Fote EC: Gait quality is improved by locomotor training in individuals with $\mathrm{SCl}$ regardless of training approach. $J$ Neuroeng Rehabil 2009, 6:36-47.

18. Swinnen E, Duerinck S, Baeyens J-P, Meeusen R, Kerckhofs E: Effectiveness of robot-assisted gait training in persons with spinal cord injury: a systematic review. J Rehabil Med 2010, 42:520-526.
19. Barbeau H, Rossignol S: Recovery of locomotion after chronic spinalization in the adult cat. Brain Res 1987, 412:84-95.

20. Dobkin $\mathrm{BH}$ : Spinal and supraspinal plasticity after incomplete spinal cord injury: correlations between functional magnetic resonance imaging and engaged locomotor networks. Prog Brain Res 2000, 128:99

21. Muir GD, Steeves JD: Sensorimotor stimulation to improve locomotor recovery after spinal cord injury. Trends Neurosci 1997, 20:72-77.

22. Winchester P, McColl R, Querry R, Foreman N, Mosby J, Tansey K, Williamson J: Changes in supraspinal activation patterns following robotic locomotor therapy in motor-incomplete spinal cord injury. Neurorehabil Neural Repair 2005, 19:313-324.

23. Colombo G, Joerg M, Dietz V: Treadmill training of paraplegic patients using a robotic orthosis. J Rehabil Res Dev 2000, 37:693-700.

24. Colombo G, Wirz M, Dietz V: Driven gait orthosis for improvement of locomotor training in paraplegic patients. Spinal Cord 2001, 39:252-255.

25. Schwartz I, Sajina A, Neeb M, Fisher I, Katz-Luerer M, Meiner Z: Locomotor training using a robotic device in patients with subacute spinal cord injury. Spinal Cord 2011, 49:1062-1067.

26. Hidler J, Wisman W, Neckel N: Kinematic trajectories while walking within the Lokomat robotic gait-orthosis. Clin Biomechanics 2008, 23:1251-1259.

27. Mirbagheri MM, Kindig M, Niu X, Varoqui D, Conaway P: Robotic-locomotor training as a tool to reduce neuromuscular abnormality in spinal cord injury: the application of system identification and advanced longitudinal modeling. IEEE Int Conf Rehabil Robot 2013, 2013:6650497.

28. Mazzoleni S, Boldrini E, Laschi C, Carrozza M, Stampacchia G, Rossi B: Changes on EMG activation in healthy subjects and incomplete $\mathrm{SCl}$ patients following a robot-assisted locomotor training. IEEE Int Conf Rehabil Robot 2011, 2011:5975467.

29. Lam T, Wirz M, Lunenburger L, Dietz V: Swing phase resistance enhances flexor muscle activity during treadmill locomotion in incomplete spinal cord injury. Neurorehabil Neural Repair 2008, 22:438-446.

30. van der Salm A, Nene AV, Maxwell DJ, Veltink PH, Hermens HJ, IJzerman MJ: Gait impairments in a group of patients with incomplete spinal cord injury and their relevance regarding therapeutic approaches using functional electrical stimulation. Artif Organs 2005, 29:8-14.

31. Dietz V, Colombo G, Jensen L, Baumgartner L: Locomotor capacity of spinal cord in paraplegic patients. Ann Neurol 1995, 37:574-582.

32. Kemoun G, Thoumie P, Boisson D, Guieu JD: Ankle dorsiflexion delay can predict falls in the elderly. J Rehabil Med 2002, 34:278-283.

33. Olney SJ, Griffin MP, McBride ID: Temporal, kinematic, and kinetic variables related to gait speed in subjects with hemiplegia: a regression approach. Phys Ther 1994, 74:872-885.

34. Olney SJ, GrifJin MP, Monga TN, McBride ID: Work and power in gait of stroke patients. Knee 1991, 1:H2.

35. Lehmann JF, Condon SM, Price R, DeLateur B: Gait abnormalities in hemiplegia: their correction by ankle-foot orthoses. Arch Phys Med Rehabil 1987, 68:763-771.

36. Gil-Agudo A, Pérez-Nombela S, Forner-Cordero A, Pérez-Rizo E, Crespo-Ruiz $B$, del Ama-Espinosa A: Gait kinematic analysis in patients with a mild form of central cord syndrome. J Neuroeng Rehabil 2011, 8:7.

37. Wirth $B$, van Hedel $\mathrm{H}$, Curt A: Foot control in incomplete $\mathrm{SCl}$ : distinction between paresis and dexterity. Neurol Res 2008, 30:52-60.

38. Hansen NL, Conway BA, Halliday DM, Hansen S, Pyndt HS, Biering-Sorensen F, Nielsen JB: Reduction of common synaptic drive to ankle dorsiflexor motoneurons during walking in patients with spinal cord lesion. J Neurophysiol 2005, 94:934-942.

39. Wirth B, van Hedel HJ, Curt A: Changes in corticospinal function and ankle motor control during recovery from incomplete spinal cord injury. J Neurotrauma 2008, 25:467-478.

40. Gorgey A, Dudley G: Skeletal muscle atrophy and increased intramuscular fat after incomplete spinal cord injury. Spinal Cord 2007, 45:304-309.

41. Shah PK, Stevens JE, Gregory CM, Pathare NC, Jayaraman A, Bickel SC, Bowden M, Behrman AL, Walter GA, Dudley GA, Vandenborne K: Lowerextremity muscle cross-sectional area after incomplete spinal cord injury. Arch Phys Med Rehabil 2006, 87:772-778.

42. Ashby P, Wiens M: Reciprocal inhibition following lesions of the spinal cord in man. J Physiol 1989, 414:145-157.

43. Yanagisawa N, Tanaka R: Reciprocal la inhibition in spastic paralysis in man. Electroencephalography and clinical neurophysiology. Electroencephalogr Clin Neurophysiol Suppl 1978, 34:521-526. 
44. Dobkin BH, Firestine A, West M, Saremi K, Woods R: Ankle dorsiflexion as an fMRI paradigm to assay motor control for walking during rehabilitation. Neuroimage 2004, 23:370-381.

45. Ashworth B: Preliminary trial of carisoprodol in multiple sclerosis. Practitioner 1964, 192:540-542.

46. Bohannon RW, Smith MB: Interrater reliability on a modified Ashworth scale of muscle spasticity. Phys Ther 1987, 67:206-207.

47. Ditunno JF Jr, Ditunno PL, Graziani V, Scivoletto G, Bernardi M, Castellano V, Marchetti M, Barbeau H, Frankel HL, D'Andrea Greve JM, Ko HY, Marshall R, Nance P: Walking index for spinal cord injury (WISCI): an international multicenter validity and reliability study. Spinal Cord 2000, 38:234-243.

48. Ditunno PL, Ditunno JF Jr: Walking index for spinal cord injury (WISCI II): scale revision. Spinal Cord 2001, 39:654-656

49. Marino RJ, Barros T, Biering-Sorensen F, Burns SP, Donovan WH, Graves DE, Haak M, Hudson LM, Priebe MM: International standards for neurological classification of spinal cord injury. J Spinal Cord Med 2003, 26:\$50.

50. Bohannon RW: Reference values for teh timed up and go test: a descriptive meta-analysis. J Geriatric Physical Therapy 2006, 29:64-68.

51. Jackson AB, Carnel CT, Ditunno JF, Read MS, Boninger ML, Schmeler MR, Williams SR, Donovan WH: Outcome measures for gait and ambulation in the spinal cord injury population. I Spinal Cord Med 2008, 31:487-499.

52. Tsao C, Mirbagheri MM: Upper limb impairments associated with spasticity in neurological disorders. J Neuroeng Rehabil 2007, 4:1-15.

53. Kepple TM, Siegel KL, Stanhope SJ: Relative contributions of the lower extremity joint moments to forward progression and support during gait. Gait Posture 1997, 6:1-8.

54. Neptune RR, Kautz S, Zajac F: Contributions of the individual ankle plantar flexors to support, forward progression and swing initiation during walking. J Biomech 2001, 34:1387-1398.

55. Jayaraman A, Shah P, Gregory C, Bowden M, Stevens J, Bishop M, Walter G, Behrman A, Vandenborne K: Locomotor training and muscle function after incomplete spinal cord injury: case series. J Spinal Cord Med 2008, 31:185.

56. Giangregorio L, McCartney N: Bone loss and muscle atrophy in spinal cord injury: epidemiology, fracture prediction, and rehabilitation strategies. J Spinal Cord Med 2006, 29:489.

57. Stewart BG, Tarnopolsky MA, Hicks AL, McCartney N, Mahoney DJ, Staron RS, Phillips SM: Treadmill training-induced adaptations in muscle phenotype in persons with incomplete spinal cord injury. Muscle Nerve 2004, 30:61-68.

58. Müller R, Dietz V: Neuronal function in chronic spinal cord injury: divergence between locomotor and flexion-and H-reflex activity. Clin Neurophysio/ 2006, 117:1499-1507.

59. Wirth B, van Hedel HJ, Curt A: Ankle paresis in incomplete spinal cord injury: relation to corticospinal conductivity and ambulatory capacity. J Clin Neurophysiol 2008, 25:210-217.

60. Thomas SL, Gorassini MA: Increases in corticospinal tract function by treadmill training after incomplete spinal cord injury. J Neurophysiol 2005, 94:2844-2855

61. Ada L, Canning CG, Low SL: Stroke patients have selective muscle weakness in shortened range. Brain 2003, 126:724-731.

62. Boorman G, Hulliger M, Lee RG, Tako K, Tanaka R: Reciprocal la inhibition in patients with spinal spasticity. Neurosci Lett 1991, 127:57-60.

63. Gómez-Soriano J, Castellote J, Pérez-Rizo E, Esclarin A, Taylor J: Voluntary ankle flexor activity and adaptive coactivation gain is decreased by spasticity during subacute spinal cord injury. Exp Neurol 2010, 224:507-516.

64. Pandyan A, Johnson G, Price $C$, Curless $R$, Barnes $M$, Rodgers $H$ : A review of the properties and limitations of the Ashworth and modified Ashworth Scales as measures of spasticity. Clin Rehabil 1999, 13:373-383.

65. Allison S, Abraham L, Petersen C: Reliability of the Modified Ashworth Scale in the assessment of plantarflexor muscle spasticity in patients with traumatic brain injury. Int J Rehabil Res 1996, 19:67-78.

66. Craven B, Morris A: Modified Ashworth scale reliability for measurement of lower extremity spasticity among patients with SCI. Spinal Cord 2010, 48:207-213.

67. Haas BM, Bergstrom E, Jamous A, Bennie A: The inter rater reliability of the original and of the modified Ashworth scale for the assessment of spasticity in patients with spinal cord injury. Spinal Cord 1996, 34:560-564.
68. Mirbagheri MM, Patel C, Quiney K: Robotic-assisted locomotor training impact on neuromuscular properties and muscle strength in spinal cord injury. Conf Proc IEEE Eng Med Biol Soc 2011, 2011:4132-4135. 2011:4132-4135.

69. Bressel E, McNair PJ: The effect of prolonged static and cyclic stretching on ankle joint stiffness, torque relaxation, and gait in people with stroke. Phys Ther 2002, 82:880-887.

70. Bensmail D, Robertson JV, Fermanian C, Roby-Brami A: Botulinum toxin to treat upper-limb spasticity in hemiparetic patients: analysis of function and kinematics of reaching movements. Neurorehabil Neural Repair 2010, 24:273-281.

71. Damiano DL, Laws E, Carmines DV, Abel MF: Relationship of spasticity to knee angular velocity and motion during gait in cerebral palsy. Gait Posture 2006, 23:1-8.

72. Latash ML, Penn RD: Changes in voluntary motor control induced by intrathecal baclofen in patients with spasticity of different etiology. Physiother Res Int 1996, 1:229-246.

73. Musselman KE: Clinical significance testing in rehabilitation research: what, why, and how? Phys Ther Rev 2007, 12:287-296.

doi:10.1186/1743-0003-11-46

Cite this article as: Varoqui et al:: Ankle voluntary movement

enhancement following robotic-assisted locomotor training in spina cord injury. Journal of NeuroEngineering and Rehabilitation 2014 11:46.

\section{Submit your next manuscript to BioMed Central and take full advantage of:}

- Convenient online submission

- Thorough peer review

- No space constraints or color figure charges

- Immediate publication on acceptance

- Inclusion in PubMed, CAS, Scopus and Google Scholar

- Research which is freely available for redistribution
C Biomed Central 